\title{
SOME GEOMETRICAL INEQUALITIES FOR SETS IN HILBERT SPACE ${ }^{1}$
}

\author{
HELMUT AULBACH
}

1. Introduction. The purpose of this paper is to introduce certain geometrical constants for sets in Hilbert space and then derive a number of inequalities among them. Some of these constants are trivially zero for compact, metric spaces, so that the noncompactness of Hilbert space plays an essential role.

We first introduce a series of constants associated with a set in Hilbert space the first of which is the transfinite diameter introduced by Fekete. ${ }^{2}$ Let the points $P_{1}, P_{2}, \cdots, P_{n+1}$ belong to a set $S$ and let $\overline{P_{i} P_{k}}$ denote the distance between $P_{i}$ and $P_{k}$. Define

$$
D_{n}(S)=\underset{P_{1}, P_{2}, \cdots, P_{n+1} \in S}{\operatorname{least} u p p e r \text { bound }}\left[\prod_{i<k} \overline{P_{i} P_{k}}\right]^{1 / C_{n+1,2}} .
$$

Later we shall define the quantities $\Delta_{n, k}$ for $n \geqq k$ of which $D_{n}(S)$ is the special case $\Delta_{n, 1}$. It will be shown that the $\Delta_{n, k}$ form a nonincreasing sequence of non-negative numbers as $n$ increases, and therefore they have a limit. The $\lim _{n \rightarrow \infty} D_{n}(S)$, denoted by $\Delta$, is the transfinite diameter of the set $S$.

To define the $\Delta_{n, k}$ we introduce the concept of the side of a simplex.

Consider an $n$-dimensional simplex $T_{n}$ : By the side of $T_{n}$, to be denoted by $s^{[n]}\left(T_{n}\right)$ or simply $s^{[n]}$, we shall mean the length of the edge of that $n$-dimensional, regular simplex whose volume equals the volume of $T_{n}$. The $n$-dimensional side of a set $S$, denoted by $s_{n}{ }^{*}(S)$ or just $s_{n}^{*}$ if there is no danger of confusion, is defined to be the least upper bound of the sides of $n$-dimensional simplices whose vertices are in $S$.

By means of a lemma, which is proved in $\$ 2$, we shall show that the $\lim _{n \rightarrow \infty} s_{n}^{*}(S)$ exists for any bounded set $S$. The lemma states: For any simplex $T_{n}$,

Presented to the Society, December 29, 1949; received by the editors October 17, 1949.

${ }^{1}$ A slight enlargement of this paper was submitted in partial fulfillment of the requirements for the Ph.D. degree at Syracuse University. The author wishes to thank Professor Charles Loewner who, with constant aid and encouragement, made this thesis possible, and the Office of Naval Research, contract number N6 ONR 248, which partially sponsored the work.

2 M. Fekete, Über den transfiniten Durchmesser ebener Punktmengen, Math. Zeit. vol. 32 (1930) pp. 108-114. 


$$
s_{i}^{[n]} \leqq\left(s_{1}^{[n-1]} \cdot s_{2}^{[n-1]} \cdots \cdots \cdot s_{n+1}^{[n-1]}\right)^{1 /(n+1)}
$$

where the $s_{i}^{[n-1]}$ are the sides of the $(n-1)$-dimensional faces of $T_{n}$.

Consider a bounded set $S$. Choose an $\epsilon>0$. Let $T_{n}$ be an $n$-dimensional simplex whose vertices are in $S$ such that

$$
s_{n}^{*}-\epsilon<s^{[n]}\left(T_{n}\right) \text {, }
$$

where $s_{n}^{*}$ is the $n$-dimensional side of $S$ and $s^{[n]}\left(T_{n}\right)$ is the side of $T_{n}$. Now,

$$
s^{[n]}\left(T_{n}\right) \leqq\left(s_{1}^{[n-1]} \cdot s_{2}^{[n-1]} \cdots \cdots \cdot s_{n+1}^{[n-1]}\right)^{1 /(n+1)} \leqq s_{n-1}^{*} .
$$

The first inequality is true because of the lemma and the second follows from the fact that each $s_{i}{ }^{[n-1]} \leqq s_{n-1}^{*}$. Hence, $s_{n}^{*}-\epsilon<s_{n-1}^{*}$.

But $\epsilon$ is arbitrary; therefore, $s_{n}^{*} \leqq s_{n-1}^{*}$. The diameter of a set $D \equiv D_{1}=s_{1}^{*} \geqq s_{2}^{*} \geqq \ldots$. This nonincreasing sequence of non-negative numbers has a non-negative limit $\sigma$ which will be called the transfinite side of $S . \sigma=\lim _{n \rightarrow \infty} s_{n}^{*}$.

Again consider $n+1$ points $P_{1}, P_{2}, \cdots, P_{n+1}$ belonging to a bounded set $S$. Define $\Delta_{n, k}(S)$ or $\Delta_{n, k}$ for $n \geqq k$ as:

$$
\Delta_{n, k}=\underset{P_{1}, P_{2}, \cdots, P_{n+1} \in S}{\text { least upper bound }}\left[s_{1}^{[k]} \cdot s_{2}^{[k]} \cdots s_{C_{n+1}, k+1}^{[k]}\right]^{1 / C_{n+1}, k+1} \text {, }
$$

where the $s_{i}^{[k]}$ are the sides of the $k$-dimensional faces of the simplex $T_{n}$ whose vertices are $P_{1}, P_{2}, \cdots, P_{n+1}$. Clearly there are $C_{n+1, k+1}$ different simplices. In order to facilitate the writing of the formulas let us denote the geometric mean of the sides of the $k$-dimensional faces of the simplex $T_{n}$ by $G_{P_{1}}^{k}, \cdots, P_{n+1}$.

To prove that $\Delta_{n, k}$ forms a nonincreasing sequence as $n$ increases, consider any $n+2$ points of $S$ and notice the equation,

$$
\begin{aligned}
& G_{P_{1}, P_{2}}^{k}, \cdots, P_{n+2} \\
& \quad=\left[G_{P_{1}, P_{2}, \cdots, P_{n+1}}^{k} \cdot G_{P_{1}}^{k} \cdots, P_{n}, P_{n+2} \cdots G_{P_{2}, P_{3}, \cdots, P_{n+2}}^{k}\right]^{1 /(n+2)} .
\end{aligned}
$$

By a suitable choice of $P_{1}, P_{2}, \cdots, P_{n+2}$, the left side of this equation can be made to differ from $\Delta_{n+1, k}$ by less than an arbitrary $\epsilon>0$. Each factor on the right side of the equation is not greater than $\Delta_{n, k}$. Therefore, $\Delta_{n+1, k}-\epsilon \leqq \Delta_{n, k}$, and since $\epsilon$ is arbitrary, $\Delta_{n+1, k} \leqq \Delta_{n, k}$.

For a bounded set, $\Delta_{k, k}$ is clearly finite. Since we have $\Delta_{k, k} \geqq \Delta_{k+1, k}$ $\geqq \cdots$, the $\lim _{n \rightarrow \infty} \Delta_{n, k}$ exists. Call it the kth transfinite diameter of the set and denote it by $\Delta_{k}$. For $k=1$ we obtain Fekete's transfinite diameter. We notice that $\Delta_{k, k}=s_{k}^{*}$ and therefore $\Delta_{k} \leqq s_{k}^{*}$.

Now to prove that $\Delta_{n, k+1} \leqq \Delta_{n, k}$ (where $n \geqq k+1$ ) for a bounded set $S$, let $P_{1}, \cdots, P_{n+1}$ belong to $S$ such that 


$$
\Delta_{n, k+1}-G_{P_{1}}^{k+1} \cdots, P_{n+1}<\epsilon
$$

where $\epsilon>0$. By application of the lemma to each term $s_{i}{ }^{[k+1]}$ comprising $G_{P_{1}}^{k+1}, \cdots, P_{n+1}$ we see that $G_{P_{1}, \cdots, P_{n+1}}^{k+1} \leqq G_{P_{1}, \cdots, P_{n+1}}^{k} \leqq \Delta_{n, k}$. In fact, by repeated use of the lemma we have the series of inequalities:

$$
G_{P_{1}}^{k}, \cdots, P_{n+1} \geqq G_{P_{1}}^{k+1} \cdots, P_{n+1} \geqq \cdots \geqq G_{P_{1}, \cdots, P_{n+1}}^{n} .
$$

But $\Delta_{n, k+1}-\epsilon<G_{P_{1}, \cdots, P_{n+1}}^{k+1}$. Hence $\Delta_{n, k+1}-\epsilon \leqq \Delta_{n, k}$, and by the arbitrariness of $\epsilon$ it follows that $\Delta_{n, k+1} \leqq \Delta_{n, k}$. But this inequality holds for all $n \geqq k+1$; therefore by making $n \rightarrow \infty$ we obtain

$$
\Delta_{k+1} \leqq \Delta_{k}
$$

Again we have a nonincreasing sequence of non-negative numbers proving the existence of their limit. Furthermore, $\lim _{k \rightarrow \infty} \Delta_{k}=\sigma$. We shall prove this by showing that $\sigma \leqq \Delta_{k}$. The proof can be obtained in the following way: Let $P_{1}, \cdots, P_{n+1}$ be points of the set so that for a given $\epsilon>0, s_{n}^{*}-\epsilon \leqq G_{P_{1}}^{n}, \cdots, P_{n+1}=s_{n}(T)$. Applying the series of inequalities (1) we see that

$$
G_{P_{1}, \cdots, P_{n+1}}^{n} \leqq G_{P_{1}, \cdots, P_{n+1}}^{k} \quad \text { for } n \geqq k .
$$

Moreover, $G_{P_{1}}^{k}, \cdots, P_{n+1} \leqq \Delta_{n, k}$; therefore, $s_{n}^{*}-\epsilon \leqq \Delta_{n, k}$. But $\epsilon$ is arbitrary. Hence $s_{n}^{*} \leqq \Delta_{n ; k}$, and taking the limit as $n \rightarrow \infty$ we obtain $\sigma \leqq \Delta_{k}$. Previously we saw that $\Delta_{k} \leqq s_{k}^{*}$. Let $k \rightarrow \infty$, then $\sigma \leqq \lim _{k \rightarrow \infty} \Delta_{k}$ $\leqq \sigma$ which proves the equality.

The last quantity to be defined was first implicitly introduced in a paper by Loewner. ${ }^{3}$ Suppose a set can be covered by a sequence of spheres whose radii tend to zero. This sequence of spheres has at least one sphere of largest radius. Now consider all possible such coverings of the given set and take the greatest lower bound of the maximum radii. This shall be called the transfinite radius of the set and is denoted by $\rho$.

The restriction that for any covering the radii should go to zero is essential. For otherwise $\rho$ would be zero for every set since Hilbert space is separable. In his paper Loewner proved that the transfinite radius of a sphere equals the radius of the sphere.

\section{The fundamental lemma.}

Lemma. For any simplex $T_{n}$

${ }^{3} \mathrm{~K}$. Loewner, Grundzilge einer Inhaltstheorie im Hilbertschen Raume, Ann. of Math. vol. 40 (1939). 


$$
s^{[n]} \leqq\left(s_{1}^{[n-1]} \cdot s_{2}^{[n-1]} \cdots \cdots \cdot s_{n+1}^{[n-1]}\right)^{1 /(n+1)}
$$

where the $s_{i}^{[n-1]}$ are the sides of the $(n-1)$-dimensional faces of $T_{n}$.

Proof. Let us show that the following extremum problem has a unique solution and let us find this solution: to find the $n$-dimensional simplex whose volume is a maximum under the restriction that the product of the volumes of the $(n-1)$-dimensional faces is a given constant $k$. In the course of the proof it becomes evident that such a maximum exists and is unique.

For any $n$-dimensional simplex $T$ let $\alpha_{i}$ be the vector whose length equals the volume of the $i$ th $(n-1)$-dimensional face, where we assume the faces to be ordered in some manner, and whose direction is the direction of the inward normal to the $i$ th face. Since the simplex is closed,

$$
\alpha_{1}+\alpha_{2}+\cdots+\alpha_{n+1}=0 \text {. }
$$

Our restriction is

$$
\left|\alpha_{1}\right| \cdot\left|\alpha_{2}\right| \cdots \cdot\left|\alpha_{n+1}\right|=k \text {. }
$$

Let $A$ be the simplex determined by the end points of $\alpha_{1}, \alpha_{2}, \cdots$, $\alpha_{n+1}$, where the $\alpha$ 's emanate from the origin.

Assume that one of the vertices of $T$ is the origin. Thus we may consider $T$ to be the simplex spanned by $n$ vectors denoted by $\beta_{1}, \beta_{2}, \cdots, \beta_{n}$.

To digress for a moment, consider the simplex determined by the $n+1$ linearly independent points $O, P_{1}, \cdots, P_{n}$. Denote vectors determining $P_{1}, \cdots, P_{n}$ which emanate from the origin by $A_{1}, \cdots, A_{n}$. We also say that the vectors $A_{1}, \cdots, A_{n}$ span the simplex. Let $A_{i}$ have components $a_{i 1}, a_{i 2}, \cdots, a_{i n}$. The volume of this simplex, denoted by $V\left(A_{1}, \cdots, A_{n}\right)$, equals $(1 / n !)\left|D\left(A_{1}, \cdots, A_{n}\right)\right|$ where $D\left(A_{1}, \cdots, A_{n}\right)$ is the determinant whose elements are the components of $A_{1}, \cdots, A_{n}$. It is well known that $V\left(A_{1}, \cdots, A_{n}\right)$ is an irreducible function of the components of $A_{1}, \cdots, A_{n}$.

Hence $V(T)$ is an irreducible polynomial of the components of $\beta_{1}, \cdots, \beta_{n}$. Moreover, since each component of an $\alpha$ is a polynomial in the components of the $\beta^{\prime}$ s, $V(A)$ is a polynomial in the components of $\beta_{1}, \cdots, \beta_{n}$. Now $V(A)=0$ if and only if $V(T)=0$ because if either of the volumes is zero it means that the dimension of that simplex is less than $n$ and therefore the other volume is zero also. And since we may think of the components as complex variables, it follows that 


$$
V(A)=c_{n} V^{q}(T),
$$

because of the theorem which states: If $f$ and $g$ are each polynomials of some complex variables, if $g$ is an irreducible function of these variables, and if $f$ and $g$ are always zero simultaneously, then $f=k_{1} g^{k_{2}}$, where $k_{2}$ is a positive integer. That $q=n-1$ follows immediately from the fact that each edge of $A$ is the volume of an $(n-1)$-dimensional face of $T$. To determine $c_{n}$, let $T$ be the simplex where the $\beta$ 's are all of length $a$ and have the directions of the first $n$ unit vectors. Then

$$
V(T)=\frac{a^{n}}{n !}, \quad \text { and } \quad V(A)=\frac{(n+1)}{n !}\left[\frac{a^{n-1}}{(n-1) !}\right]^{n}
$$

Hence

$$
V(A)=\frac{(n+1) n^{n}}{(n !)^{2}} V^{n-1}(T), \quad \text { and } \quad c_{n}=\frac{(n+1) n^{n}}{(n !)^{2}}
$$

Therefore, to find the maximum $V(T)$ it is sufficient to find the maximum $V(A)$.

Let $\alpha_{i}=a_{i} \epsilon_{i}$, where $a_{i}>0$ and $\left|\epsilon_{i}\right|=1$ for $i=1,2, \cdots, n+1$. By considering any $n$ of the $\alpha$ 's we obtain a volume equal to $V(A) /(n+1)$.

$$
\begin{aligned}
& \frac{V(A)}{n+1}=\frac{1}{n !}\left|D\left(\alpha_{i}, \cdots, \alpha_{i-1}, \alpha_{i+1}, \cdots, \alpha_{n+1}\right)\right| \\
& \quad=\frac{a_{1} \cdots a_{i-1} \cdot a_{i+1} \cdots \cdots a_{n+1}}{n !}\left|D\left(\epsilon_{1}, \cdots, \epsilon_{i-1}, \epsilon_{i+1}, \cdots, \epsilon_{n+1}\right)\right|,
\end{aligned}
$$

where $i$ ranges from 1 to $n+1$. Multiplying these $n+1$ equations together we obtain,

$$
\begin{aligned}
{\left[\frac{V(A)}{n+1}\right]^{n+1}=\frac{\left(a_{1} \cdot a_{2} \cdots \cdots a_{n+1}\right)^{n}}{(n !)^{n+1}}\left|D\left(\epsilon_{1}, \cdots, \epsilon_{n}\right)\right| } & \cdots \\
& \cdot\left|D\left(\epsilon_{2}, \cdots, \epsilon_{n+1}\right)\right| .
\end{aligned}
$$

Since the geometric mean is not greater than the arithmetic mean we have

$$
\begin{aligned}
& {\left[\frac{V(A)}{n+1}\right]^{n+1}} \\
& \quad \leqq \frac{k^{n}}{(n !)^{n+1}}\left[\frac{\left|D\left(\epsilon_{1}, \cdots, \epsilon_{n}\right)\right|+\cdots+\left|D\left(\epsilon_{2}, \cdots, \epsilon_{n+1}\right)\right|}{n+1}\right]^{n+1},
\end{aligned}
$$

or 


$$
V(A) \leqq \frac{k^{n /(n+1)}}{n !}\left[\left|D\left(\epsilon_{1}, \cdots, \epsilon_{n}\right)\right|+\cdots+\left|D\left(\epsilon_{2}, \cdots, \epsilon_{n+1}\right)\right|\right] .
$$

The sum $\left|D\left(\epsilon_{1}, \cdots, \epsilon_{n}\right)\right|+\cdots+\left|D\left(\epsilon_{2}, \cdots, \epsilon_{n+1}\right)\right|$ represents the volume of the simplex spanned by the vectors $\epsilon_{1}, \cdots, \epsilon_{n+1}$. It is easy to show that the volume spanned by any number of unit vectors is a maximum if the end points of the unit vectors form a regular simplex. If we imagine the end points of $\epsilon_{1}, \cdots, \epsilon_{n+1}$ to have variable nonnegative weights attached to them whose sum is one, then any point in the convex closure of the end points of $\epsilon_{1}, \cdots, \epsilon_{n+1}$ can be represented uniquely as a linear combination of $\epsilon_{1}, \cdots, \epsilon_{n+1}$. The point $O$ can be represented by $\epsilon_{1}+\epsilon_{2}+\cdots+\epsilon_{n+1}$. But $0=a_{1} \epsilon_{1}+\cdots$ $+a_{n+1} \epsilon_{n+1}$. Therefore, all $a_{i}$ are equal. This means

$$
\left|\alpha_{1}\right|=\left|\alpha_{2}\right|=\cdots=\left|\alpha_{n+1}\right|=k^{1 /(n+1)}
$$

because of (2). Moreover, since the $\alpha_{i}$ have the directions of the $\epsilon_{i}$, the simplex they form is regular. And if $A$ is a regular simplex, so is $T$. Therefore, the simplex of maximum volume having the product of the volumes of its $(n-1)$-dimensional faces equal to a given constant is regular.

In the case of a regular simplex $R$, the side of $R$ equals the side of any face; therefore, in particular,

$$
s_{R}^{[n]}=\left(s_{1}^{[n-1]} \cdot s_{2}^{[n-1]} \cdots \cdots \cdot s_{n+1}^{[n-1]}\right)^{1 /(n+1)} .
$$

Since the product of the volumes of the $(n-1)$-dimensional faces of $T$ equals a given constant so does the product of the sides of these faces. But for $R, s^{[n]}$ is a maximum; therefore, for any simplex $T$

$$
s^{[n]} \leqq\left(s_{1}^{[n-1]} \cdot s_{2}^{[n-1]} \cdots \cdots \cdot s_{n+1}^{[n-1]}\right)^{1 /(n+1)},
$$

and the equality holds only if the simplex is regular.

COROLLARY. By making repeated use of this inequality; that is, by applying this inequality to the $(n-1)$-dimensional simplices which are the faces of $T$, then applying this lemma again to the $(n-2)$-dimensional simplices, and so on, we obtain the inequality:

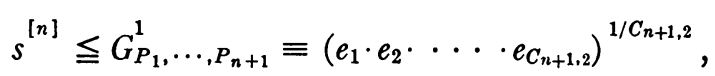

where the $e_{i}$ are the edges of $T$.

\section{The fundamental theorem.}

Theorem 1. For any bounded set, $\rho \leqq \sigma / 2^{1 / 2}$. 
Proof. Let $S$ be a bounded set. For any $\epsilon>0$ we can find points $P_{1}, \cdots, P_{n+1}$ belonging to $S$ which form an $n$-dimensional simplex $T_{n}$ so that

$$
V\left(T_{n}\right) \geqq V_{n}^{*}-\epsilon,
$$

where $V_{n}^{*}$ is the least upper bound of the volumes of all $n$-dimensional simplices whose vertices are in $S$. Choose any other point $P$ of $S$ whose height is $h_{n}$ from the lowest-dimensional hyperplane $H$ through $P_{1}, \cdots, P_{n+1}$. The vertices of $T_{n}$ together with $P$ form an $(n+1)$-dimensional simplex $T_{n+1}$. The volume of any $n$-dimensional face of $T_{n+1}$ is not greater than $V_{n}^{*}$.

An isoperimetric theorem states that the maximum volume of a simplex of given surface area is achieved if the simplex is regular. Clearly, the larger the surface area the larger the maximum volume.

Let $R$ denote the regular, $(n+1)$-dimensional simplex each of whose $n$-dimensional faces has volume $V_{n}^{*}$. Now $V\left(T_{n+1}\right) \leqq V_{n+1}^{*}$, where $V_{n+1}^{*}$ is defined analogously to $V_{n}^{*}$. And $V_{n+1}^{*} \leqq V(R)$, because the surface area of the simplex whose volume equals $V_{n+1}^{*}$ is not greater than the surface area of $R$.

$$
\begin{aligned}
V\left(T_{n+1}\right) & =\frac{1}{n+1} \cdot V\left(T_{n}\right) \cdot h_{n}, \\
V(R) & =\frac{1}{n+1} \cdot V_{n}^{*} \cdot s_{n}^{*}\left(\frac{n+2}{2(n+1)}\right)^{1 / 2} .
\end{aligned}
$$

The last equation is true because the height of a regular $(n+1)$-dimensional simplex whose edge is $e$ equals $e((n+2) / 2(n+1))^{1 / 2}$, and the edge of $R$ is the same as the side of a face of $R$ which is $s_{n}^{*}$. Since

$$
V\left(T_{n+1}\right) \leqq V(R) \text { and } V_{n}^{*}-\epsilon \leqq V\left(T_{n}\right),
$$

it follows that

$$
\frac{1}{n+1}\left(V_{n}^{*}-\epsilon\right) h_{n} \leqq \frac{1}{n+1} V_{n}^{* *} s_{n}\left(\frac{n+2}{2(n+1)}\right)^{1 / 2}
$$

Therefore,

$$
h_{n} \leqq\left(1+\frac{\epsilon}{V^{*}-\epsilon}\right) s_{n}^{*}\left(\frac{n+2}{2(n+1)}\right)^{1 / 2} .
$$

Since $P$ was an arbitrary point of the set, it follows that the set must lie within the cylinder of radius $r=\left(1+\epsilon /\left(V_{n}^{*}-\epsilon\right)\right) s_{n}^{*}((n+2) / 2(n+1))^{1 / 2}$ 
having $H$ as axis. The projection of $S$ on $H$ is bounded because $S$ is bounded. Clearly, a finite number of spheres with centers in this projection and radii equal to $r+\epsilon^{\prime}$, where $\epsilon^{\prime}>0$, contain $S$. Therefore, $\rho \leqq r+\epsilon^{\prime}$ and because $\epsilon^{\prime}$ is arbitrary, $\rho \leqq r$. Also because of the arbitrariness of $\epsilon, \rho \leqq s_{n}^{*}((n+2) / 2(n+1))^{1 / 2}$. Taking the limit as $n \rightarrow \infty$, we have $\rho \leqq \sigma / 2^{1 / 2}$.

Corollary. From the inequalities established in $\$ 1$ we immediately obtain

$$
\rho \leqq \frac{\Delta_{k}}{2^{1 / 2}} \text { for } \quad k=1,2, \cdots \quad \text { and } \rho \leqq \frac{D}{2^{1 / 2}}
$$

The inequality $\rho \leqq \sigma / 2^{1 / 2}$ is the best possible because equality is attained in the case of a sphere, because for a sphere of radius $r$, $\rho=r$ and $s_{n}^{*}=(2(n+1) / n)^{1 / 2} r$. Taking the limit as $n \rightarrow \infty$, we see that $\sigma=2^{1 / 2} r$. Another set for which we have not only $\rho=\sigma / 2^{1 / 2}$ but also $\rho=D / 2^{1 / 2}$ is the simplex $T$ defined as follows: Let the origin and the end points of the first $n$ unit vectors define a simplex $T_{n}$. The closure of the limit of $T_{n}$ when $n \rightarrow \infty$ defines a simplex $T$. It can be shown that $T$ consists of the set of points $\left(x_{1}, x_{2}, \cdots\right)$ where all $x_{i} \geqq 0$ and $\sum x_{i} \leqq 1$. Clearly $D=2^{1 / 2}$, and it can be proved that $\rho=1$.

4. Compact sets. All quantities considered such as $\rho, \sigma, \Delta_{i}$ do not change if a set is replaced by its closure. It is therefore no loss of generality if we restrict ourselves to closed sets, and we shall give a characterization of the compact sets among them as expressed in the following theorem.

THEOREM 2. A necessary and sufficient condition for a closed set to be compact is that it be bounded and its transfinite side be zero.

(a) Necessity. Let $S$ be a compact set. Clearly $S$ must be bounded. To prove $\sigma=0$ we note the fact that a compact set may be mapped into a set in a finite-dimensional hyperplane by moving each point by less than a given $\epsilon>0$. Let $S$ be mapped into $S^{\prime}$ contained in the $k$-dimensional hyperplane $H_{k}$ by such an $\epsilon$-transformation. Hence $S$ lies in a cylinder of radius $\epsilon$ about $H_{k}$ as axis. We may imagine $S$ to lie in some bounded portion $C$ of the cylinder.

Consider a $(k+1)$-dimensional simplex $T$ with vertices in $S$ whose side differs by less than $\epsilon$ from the $(k+1)$-dimensional side of $S$ $\left(s_{k+1}^{*}-\epsilon \leqq s_{T}^{[k+1]}\right)$. Project $T$ into a $k$-dimensional hypersphere which is a subset of the base of the cylinder $C$ which is a hypersphere of radius $\epsilon$. This projection consists of one or the union of two $k$-dimensional simplices each of which has a volume not greater than the 
maximum volume of a $k$-dimensional simplex inscribed in a sphere of radius $\epsilon$.

Now the volume of a regular $k$-dimensional simplex of side $a$ equals

$$
\left(\frac{a}{2^{1 / 2}}\right)^{k} \frac{(k+1)^{1 / 2}}{k !} \text {. }
$$

Since the simplex of maximum volume that can be inscribed in a sphere is regular, the maximum volume of a $k$-dimensional simplex inscribed in a sphere of radius $\epsilon$ equals

$$
\left(\frac{(2(k+1) / k)^{1 / 2} \epsilon}{2^{1 / 2}}\right)^{k} \frac{(k+1)^{1 / 2}}{k !} .
$$

By the preceding considerations we have

$$
\begin{aligned}
& \left(\frac{s_{T}{ }^{[k+1]}}{2^{1 / 2}}\right)^{k+1} \frac{(k+2)^{1 / 2}}{(k+1) !} \\
& \quad \leqq 2\left\{\frac{1}{k+1} \cdot\left[\left(\frac{(2(k+1) / k)^{1 / 2} \epsilon}{2^{1 / 2}}\right)^{k} \frac{(k+1)^{1 / 2}}{k !}\right] \cdot D\right\},
\end{aligned}
$$

where $D$ is the diameter of the set. Solving for $s_{T}^{[k+1]}$ we obtain

$$
s_{T}^{[k+1]} \leqq 2^{1 / 2}\left(2 D \frac{(k+1)^{1 / 2}}{(k+2)^{1 / 2}}\right)^{1 /(k+1)}\left(\frac{(k+1)^{1 / 2}}{k^{1 / 2}}\right)^{k /(k+1)} \epsilon^{k /(k+1)} .
$$

Hence

$$
s_{k+1}^{*}-\epsilon \leqq 2^{1 / 2}\left(2 D \frac{(k+1)^{1 / 2}}{(k+2)^{1 / 2}}\right)^{1 /(k+1)}\left(\left(\frac{k+1}{k}\right)^{1 / 2}\right)^{k /(k+1)} \epsilon^{k /(k+1)}
$$

Also,

$$
\sigma \leqq 2^{1 / 2}\left(2 D \frac{(k+1)^{1 / 2}}{(k+2)^{1 / 2}}\right)^{1 /(k+1)}\left(\left(\frac{k+1}{k}\right)^{1 / 2}\right)^{k /(k+1)} \epsilon^{k /(k+1)}+\epsilon .
$$

But this inequality holds for all sufficiently large values of $k$. Let $k \rightarrow \infty$, then

$$
\sigma \leqq 2^{1 / 2} \epsilon+\epsilon .
$$

Since $\epsilon$ was arbitrary, $\sigma=0$.

(b) Sufficiency. Let $S$ be a closed bounded set and have $\sigma=0$. If $S$ is finite-dimensional, it is compact; therefore, assume $S$ to be nonfinite-dimensional. Choose $n+1$ points of $S$ forming an $n$-dimensional simplex $T_{n}$ whose volume $V_{n}$ differs from the least upper bound, 
$V_{n}^{*}$, of the volumes of $n$-dimensional simplices with vertices in $S$, by less than a given $\epsilon>0$. Choose any other point $P$ of $S$. Denote its distance from the hyperplane $H_{n}$ determined by the vertices of $T_{n}$ by $h$. To find an upper bound for $h$, notice that the simplex formed by the vertices of $T_{n}$ together with $P$ has a volume not greater than the least upper bound of the volumes of the $(n+1)$-dimensional simplices whose vertices are in $S$.

$$
\begin{gathered}
\frac{1}{n+1} V_{n} \cdot h \leqq\left(\frac{s_{n+1}^{*}}{2^{1 / 2}}\right)^{n+1} \frac{(n+2)^{1 / 2}}{(n+1) !}, \\
\frac{h}{n+1}\left[\left(\frac{s_{n}^{*}}{2^{1 / 2}}\right)^{n} \frac{(n+1)^{1 / 2}}{n !}-\epsilon\right]<\left(\frac{s_{n+1}^{*}}{2^{1 / 2}}\right)^{n+1} \frac{(n+2)^{1 / 2}}{(n+1) !} \\
h<\frac{\left(\frac{s_{n+1}^{*}}{2^{1 / 2}}\right)^{n+1} \frac{(n+2)^{1 / 2}}{n !}}{\left(\frac{s_{n}^{*}}{2^{1 / 2}}\right)^{n} \frac{(n+1)^{1 / 2}}{n !}-\epsilon} \leqq \frac{\left(\frac{s_{n}^{*}}{2^{1 / 2}}\right)^{n+1} \frac{(n+2)^{1 / 2}}{n !}}{\left(\frac{s_{n}^{*}}{2^{1 / 2}}\right)^{n} \frac{(n+1)^{1 / 2}}{n !}-\epsilon} \equiv q_{n} .
\end{gathered}
$$

Any point in $P$ of $S$ lies within a cylinder of radius $q_{n}$ with axis $H_{n}$. But $q_{n}$ can be made to differ from

$$
q_{n}^{\prime} \equiv \frac{s_{n}^{*}}{2^{1 / 2}}\left(\frac{n+2}{n+1}\right)^{1 / 2}
$$

by as little as we please. Let $n \rightarrow \infty$, then $q_{n}^{\prime} \rightarrow 0$ because $s_{n}{ }^{*} \rightarrow 0$. Hence the set may be included in a cylinder of arbitrarily small radius about a finite-dimensional hyperplane. As is well known, this is enough to show that the set is compact.

\section{BIBLIOGRAPHY}

1. M. Fekete, Über den transfiniten Durchmesser ebener Punktmengen, Math. Zeit. vol. 32 (1930).

2. K. Loewner, Grundzige einer Inhaltstheorie im Hilbertschen Raume, Ann. of Math. vol. 40 (1939).

\section{SyraCUSE UNIVERSITY}

